OPEN ACCESS

Edited by:

Leo Marcelis,

Wageningen University and

Research, Netherlands

Reviewed by:

Thomas D. Sharkey

Michigan State University,

United States

Qingwu Meng,

University of Delaware, United States

${ }^{*}$ Correspondence:

Shuyang Zhen

shuyang.zhen@tamu.edu

Specialty section: This article was submitted to Crop and Product Physiology, a section of the journal

Frontiers in Plant Science

Received: 11 April 2021 Accepted: 20 May 2021

Published: 22 June 2021

Citation:

Zhen $S$, van lersel $M$ and Bugbee $B$ (2021) Why Far-Red Photons Should

Be Included in the Definition of

Photosynthetic Photons and the Measurement of Horticultural Fixture Efficacy. Front. Plant Sci. 12:693445. doi: 10.3389/fpls.2021.693445

\section{Why Far-Red Photons Should Be Included in the Definition of Photosynthetic Photons and the Measurement of Horticultural Fixture Efficacy}

\author{
Shuyang Zhen ${ }^{1 *}$, Marc van lersel ${ }^{2}$ and Bruce Bugbee ${ }^{3}$ \\ ${ }^{1}$ Department of Horticultural Sciences, Texas A\&M University, College Station, TX, United States, ${ }^{2}$ Department of \\ Horticulture, University of Georgia, Athens, GA, United States, ${ }^{3}$ Department of Plants, Soils and Climate, Utah State \\ University, Logan, UT, United States
}

Keywords: far-red photons, fixture efficacy, photosynthesis, Emerson Enhancement Effect, extended photosynthetically active radiation

\section{INTRODUCTION}

Photons above $700 \mathrm{~nm}$ have minimal photosynthetic activity when applied alone (Emerson and Lewis, 1943; McCree, 1971) and have thus been excluded from the definition of photosynthetically active radiation (PAR; 400 to $700 \mathrm{~nm}$ ). However, those longer-wavelength photons have synergistic activity with photons in the PAR range (Emerson et al., 1957). Recent studies using lasers and LEDs with narrow-band spectra have provided new insights into the photosynthetic value of farred photons (here defined as 700 to $750 \mathrm{~nm}$ ). Far-red photons preferentially excite photosystem I (Zhen and van Iersel, 2017), at wavelengths at least up to $732 \mathrm{~nm}$ (Zhen et al., 2019). In crop-plant communities, far-red photons elicit photosynthetic activity equal to PAR photons when delivered at up to $30 \%$ of the total photon flux (Zhen and Bugbee, 2020a). The quantum yield of plant canopies (per 400 to $750 \mathrm{~nm}$ photons) is similar under blue + red or white LEDs with and without a 15\% far-red photon substitution (Zhen and Bugbee, 2020b). The definition of photosynthetic photons, and efficacy measurements of horticultural fixtures, need to include far-red photons because this extended range (referred to as ePAR) better predicts photosynthesis.

\section{HISTORICAL BACKGROUND}

Photosynthesis has long been known to be wavelength-dependent (Hoover, 1937; Emerson and Lewis, 1943). At low photon flux densities, McCree (1971) and Inada (1976) found that red photons $(600-700 \mathrm{~nm})$ drive photosynthesis more efficiently than green $(500-600 \mathrm{~nm})$, followed by blue $(400-500 \mathrm{~nm})$ photons. Because green photons penetrate deeper into leaves, more recent studies indicate that at higher photon flux densities red and green photons are used more efficiently than blue photons (Terashima et al., 2009; Liu and van Iersel, 2021). Longer-wavelength far-red photons (above $700 \mathrm{~nm}$ ), on the other hand, are largely inactive for photosynthesis when applied alone (Emerson and Lewis, 1943; McCree, 1971) and have thus been excluded from the definition of photosynthetically active radiation (PAR; $400-700 \mathrm{~nm}$ ).

The rapid decline in photosynthetic efficiency at longer wavelengths (above $\sim 685 \mathrm{~nm}$ ) was first observed by Emerson and Lewis (1943) in green algae (the "red drop"). Over a decade later, the same research group found that the photosynthetic rate under simultaneous illumination with photons above $680 \mathrm{~nm}$ and shorter-wavelength light was greater than the sum of the rates from applying 
each light separately (Emerson et al., 1957). This is now known as the Emerson Enhancement Effect. This enhancement effect among shorter- and longer-wavelength photons was later found to be due to the distinct excitation spectra of the two photosystems-PSI and PSII (Hill and Bendall, 1960; Duysens and Amesz, 1962). While the discovery of the Emerson Enhancement Effect contributed to the identification of PSI and PSII, the significance of the wavelength synergy in photosynthetic efficiency received little attention over the next 50 years and the spectral effects on photosynthesis continued to be studied under monochromatic lights. The main reason for this oversight is the belief that only the photosynthetic efficiency of longerwavelength photons ( $680 \mathrm{~nm}$ up to $720 \mathrm{~nm}$ ) was improved by the supplementation with shorter-wavelength light, rather than a two-way synergistic interaction in which shorter- and longer-wavelength photons improve each other's photosynthetic efficiency; thus, the practical impact of the enhancement effect on photosynthesis was thought to be largely insignificant (Emerson et al., 1957; Myers and Graham, 1963; McCree, 1972a). As a result, the now widely accepted definition of PAR was developed without taking account of synergistic effects on PSI and PSII photochemistry between far-red and shorterwavelength photons.

This $400-700 \mathrm{~nm}$ range was recommended by McCree (1972b) from among the most popular definitions of PAR in use at the time. He concluded that the photon flux density between 400 and $700 \mathrm{~nm}$ was "an acceptable definition of photosynthetic flux" for nine commonly used broadspectrum lights. Interestingly, he found that photosynthetic rates, normalized based on PAR, were highest under a high-pressure sodium light (with most of the light in the red part of the spectrum) and a quartz-iodine light (rich in red and far-red photons). None of the definitions of PAR analyzed by McCree accounted for photons with wavelengths $>710 \mathrm{~nm}$. His study did not test whether including far-red photons in the definition of PAR would improve the correlation with the photosynthetic rate.

\section{RECENT STUDIES INDICATE THAT THE CLASSIC DEFINITION OF PAR NEEDS TO BE REVISED}

Recent advances in light-emitting diode (LED) technology have enabled researchers to re-visit the Emerson Enhancement Effect and study not only the short-term photosynthetic responses of single leaves under low light but also the long-term responses of plant canopies under higher photon flux densities. Zhen and van Iersel (2017) found that adding supplemental far-red photons from LEDs (peak at $735 \mathrm{~nm}$ ) to red+blue or white LED light synergistically increased the quantum yield of PSII and leaf photosynthetic rate over a wide range of light intensities (also see Murakami et al., 2018). The enhancement was slightly larger under the red/blue background light than under a warm white LED, probably because the warm white LED light already had $4 \%$ far-red photons.

Zhen et al. (2019) studied the effects of photons from 678 to $752 \mathrm{~nm}$ using laser diodes that had a narrow spectral output [full width at half maximum (FWHM) of $2-3 \mathrm{~nm}$ ]. As the wavelength of the photons increased from 678 to $703 \mathrm{~nm}$, they increasingly excited PSI more efficiently than PSII. Photons up to $732 \mathrm{~nm}$ significantly enhanced photosynthetic efficiency by exciting PSI, but photons above $752 \mathrm{~nm}$ were not effective. There was a gap between 732 and $752 \mathrm{~nm}$ because laser diodes were not available in this region.

In a subsequent study, Zhen and Bugbee (2020a) measured the net photosynthetic rate of plant canopies (including leaves, stems, roots; with communities of plants inside $100 \mathrm{~L}$ gas exchange chambers) in 14 diverse species and found that photons from far-red LEDs (700-750 nm; peak at $735 \mathrm{~nm}$ ) were as effective as traditional $400-700 \mathrm{~nm}$ photons when applied at up to $\sim 30 \%$ of the total photon flux. As expected, far-red photons alone were not effective. Additional far-red photons applied at more than $30 \%$ of the total photon flux did not result in further increases in photosynthetic rate. The photosynthetic response to increasing far-red photon flux likely saturates, because under a light composed of mostly shorter-wavelength photons that over-excites PSII, only a certain number of far-red photons are needed to restore the excitation balance between PSI and PSII. An enhancement effect only occurs up to the point at which this balance is reached.

Zhen and Bugbee (2020b) followed this study with a longterm study with lettuce grown under either blue+red or white LEDs, each with and without $15 \%$ far-red photons from farred LEDs. The total photon flux from 400 to $750 \mathrm{~nm}$ was equal among spectral treatments. Photon capture and canopy gas exchange were continuously measured, which allowed the analysis of canopy quantum yield (CQY; moles of $\mathrm{CO}_{2}$ fixed per mole of absorbed photons). CQY was equal among treatments from planting to harvest, confirming the important role of far-red photons for photosynthesis.

We have studied the effects of far-red photons at the photosystem, leaf, and canopy level, with consistent results at all three scales. Collectively, these findings provide compelling evidence for the photosynthetic value of far-red photons when combined with shorter-wavelength photons, as long as the far-red flux does not exceed about $30 \%$ of the total photon flux. This is equal to or higher than the fraction of far-red photons in sunlight under which plants have evolved.

Unfortunately, the Design Lights Consortium (2021), following ASABE Standard S640 (2017), recently decided to not expand the definition of PAR to include far-red photons, despite clear evidence of the photosynthetic efficacy of those photons. Design Lights Consortium kindly cites our research in their decision, but we do not believe that our research was interpreted accurately. One concern raised by Design Lights Consortium (2021) is that the enhancement effect may depend on the spectrum of the background light. As we explained earlier, the larger enhancement effect of far-red photons with red+blue background light compared to that under a white background light was most likely because the white light already had $~ 4 \%$ far-red photons. In addition to this, Zhen and Bugbee (2020b) found that the value of far-red photons was equal with either blue + red or white LEDs (contained $\sim 1 \%$ far-red photons). As far as we know there is no experimental evidence suggesting that 
the spectral composition in the $400-700 \mathrm{~nm}$ range affects the magnitude of the enhancement effect.

Design Lights Consortium also argued that the data presented in Zhen and van Iersel (2017) suggested that the enhancement response diminished at higher background light intensities, thus the response is non-linear and the prediction of photosynthesis with the extended PAR range would be difficult. This was a misinterpretation, since Zhen and van Iersel (2017) added the same intensity of far-red photons to varying background light intensities between 400 and $700 \mathrm{~nm}$ and found that the absolute increase in net photosynthetic rate was similar at all background light intensity levels; it was the percent increase in net photosynthetic rate that diminished at higher background light intensities, which is expected.

In addition, the Design Lights Consortium (2021) also mistakenly described the equal canopy photosynthetic rate elicited by far-red substitution in Zhen and Bugbee (2020a) as an "additive" response without any enhancement effect. In fact, the data clearly show that the far-red photons alone had little photosynthetic activity. However, when added to a background of $400-700 \mathrm{~nm}$ photons, far-red photons increased photosynthesis equal to the addition of traditional PAR photons.

Lastly, the Design Lights Consortium (2021) raised concern about the spectral response of far-red photons. Zhen et al. (2019) found that photons with wavelengths from $\sim 700$ to $732 \mathrm{~nm}$ were similarly effective in enhancing photochemical efficiency, but photons above $752 \mathrm{~nm}$ were not effective. Zhen and Bugbee (2020a) added photons from three far-red LEDs with peak output at 711,723 , and $746 \mathrm{~nm}$, respectively, to a background of red+blue light and found that the increase in photosynthesis in response to the added far-red decreased with increasing peak wavelength of the far-red LEDs. About $29 \%$ of the total photons emitted by the $711 \mathrm{~nm}$-centered LED was below $700 \mathrm{~nm}$, and about $24 \%$ of the total photons emitted by the $746 \mathrm{~nm}$-centered LED was above $750 \mathrm{~nm}$. Since these LEDs had a relatively broad emission spectrum (FWHM ranging from 18 to $24 \mathrm{~nm}$ ), it is difficult to draw a definitive conclusion on the importance of the spectral effect of far-red photons. The argument that precise knowledge of the spectral response of photosynthesis to farred photons is required before far-red can be included in the definition of PAR is inconsistent with the current definition of PAR: it is well-known that photons of different wavelengths within the $400-700 \mathrm{~nm}$ range elicit different photosynthetic responses. That has not stopped the widespread adoption of photons within the $400-700 \mathrm{~nm}$ range as the definition of PAR. Defining PAR as the photon flux density between 400 and

\section{REFERENCES}

ASABE Standard S640. (2017) Quantities and Units of Electromagnetic Radiation for Plants (Photosynthetic Organisms). Available online at: https://elibrary. asabe. org/abstract.asp?aid $=48303$

Design Lights Consortium (2021). Limitations of Predicting Far-Red's Effect on Photosynthesis. Available online at: https://www.designlights.org/default/ assets/File/Horticultural/DLC_Horticultural-Lighting-Resources_Far-RedEffect-Photosynthesis.pdf
$750 \mathrm{~nm}$ may not be perfect, but will more accurately reflect the photosynthetic activity of photons and better correlate with plant growth than the current definition. To keep the traditional PAR definition and the new definition separate, we have begun using the term extended PAR (ePAR) to refer to the sum of photons between 400 and $750 \mathrm{~nm}$.

Since the efficacy of horticultural fixtures ( $\mu$ moles of photons per joule) is calculated as photosynthetic photons/energy use, the efficacy of fixtures that include far-red photons will be counted as lower than fixtures that do not emit far-red photons. This occurs with lower color temperature white LEDs and especially fixtures with far-red LEDs. Fixture efficacy is used by energy efficiency programs to determine fixture eligibility for rebate or incentive programs. Until the definition of PAR is expanded to include farred photons (ePAR, 700-750 nm), lighting manufacturers will be discouraged from including far-red LEDs in fixtures. Changing the definition from PAR to ePAR and including photons from 400 to $750 \mathrm{~nm}$ will facilitate the development of fixtures with higher efficacy and encourage innovation (Kusuma et al., 2020). As more supporting data from more laboratories becomes available, we expect that the ePAR definition will replace the current definition of PAR. The ePAR definition should indicate that the far-red fraction cannot exceed about $30 \%$ of the total photon flux from 400 to $750 \mathrm{~nm}$ or $40 \%$ of the photon flux from 400 to $700 \mathrm{~nm}$.

\section{PRACTICAL LIMITATIONS}

Far-red photons typically cause significant stem, leaf, and/or petiole elongation, which will likely limit the maximum fraction of far-red photons to less than about $20 \%$ of the total photon flux for most crops. Because of these powerful effects, we recommend that LED manufacturers clearly indicate the fraction of far-red photons in fixture specifications $[(700-750 \mathrm{~nm}) /(400-750 \mathrm{~nm})]$.

\section{AUTHOR CONTRIBUTIONS}

This opinion piece originated from discussions among all authors. SZ wrote the first draft with input from MvI and BB. All authors revised the manuscript and have approved the manuscript.

\section{FUNDING}

This work was supported by the NASA-CUBES project award number NNX17AJ31G and by the USDA-NIFA-SCRI award number 2018-51181-28365 (LAMP Project). doi: 10.1016/0006-3002(62)90735-7

Emerson, R., Chalmers, R., and Cederstrand, C. (1957). Some factors influencing the long-wave limit of photosynthesis. Proc. Natl. Acad. Sci. U.S.A. 43:133. doi: 10.1073/pnas.43.1.133

Emerson, R., and Lewis, C. M. (1943). The dependence of the quantum yield of Chlorella photosynthesis on wave length of light. Am. J. Botany 30, 165-178. doi: 10.1002/j.1537-2197.1943.tb14744.x 
Hill, R., and Bendall, F. (1960). Function of the two cytochrome components in chloroplasts: a working hypothesis. Nature 186, 136-137. doi: $10.1038 / 186136 \mathrm{a} 0$

Hoover, W. H. (1937). The dependence of carbon dioxide assimilation in a higher plant on wavelength of radiation. Smithsonian Inst. Misc. Collect 95, 1-13.

Inada, K. (1976). Action spectra for photosynthesis in higher plants. Plant Cell Physiol. 17, 355-365.

Kusuma, P., Pattison, M., and Bugbee, B. (2020). From physics to fixtures to food: current and potential LED efficacy. Nat. Horticul. Res. 7:56. doi: 10.1038/s41438-020-0283-7

Liu, J., and van Iersel, M. W. (2021). Photosynthetic physiology of blue, green, and red light: light intensity effects and underlying mechanisms. Front. Plant Sci. 12:619987. doi: 10.3389/fpls.2021.619987

McCree, K. J. (1971). The action spectrum, absorptance and quantum yield of photosynthesis in crop plants. Agric. Meteorol. 9, 191-216. doi: 10.1016/0002-1571(71)90022-7

McCree, K. J. (1972a). Significance of enhancement for calculations based on the action spectrum for photosynthesis. Plant Physiol. 49, 704-706. doi: $10.1104 /$ pp.49.5.704

McCree, K. J. (1972b). Test of current definitions of photosynthetically active radiation against leaf photosynthesis data. Agric. Meteorol. 10, 443-453. doi: 10.1016/0002-1571(72)90045-3

Murakami, K., Matsuda, R., and Fujiwara, K. (2018). A mathematical model of photosynthetic electron transport in response to the light spectrum based on excitation energy distributed to photosystems. Plant Cell Physiol. 59, 1643-1651. doi: 10.1093/pcp/pcy085

Myers, J., and Graham, J. R. (1963). Enhancement in chlorella. Plant Physiol. 38, 105-116. doi: 10.1104/pp.38.1.105

Terashima, I., Fujita, T., Inoue, T., Chow, W. S., and Oguchi, R. (2009). Green light drives leaf photosynthesis more efficiently than red light in strong white light: revisiting the enigmatic question of why leaves are green. Plant Cell Physiol. 50, 684-697. doi: 10.1093/pcp/pcp034
Zhen, S., and Bugbee, B. (2020a). Far-red photons have equivalent efficiency to traditional photosynthetic photons: implications for redefining photosynthetically active radiation. Plant Cell Environ. 43, 1259-1272. doi: $10.1111 /$ pce. 13730

Zhen, S., and Bugbee, B. (2020b). Substituting far-red for traditionally defined photosynthetic photons results in equal canopy quantum yield for $\mathrm{CO}_{2}$ fixation and increased photon capture during long-term studies: implications for re-defining PAR. Front. Plant Sci. 11:1433. doi: 10.3389/fpls.2020. 581156

Zhen, S., Haidekker, M., and van Iersel, M. W. (2019). Far-red light enhances photochemical efficiency in a wavelength-dependent manner. Physiol. Plantarum 167, 21-33. doi: 10.1111/ppl.12834

Zhen, S., and van Iersel, M. W. (2017). Far-red light is needed for efficient photochemistry and photosynthesis J. Plant Physiol. 209, 115-122. doi: 10.1016/j.jplph.2016.1 2.004

Disclaimer: Any opinions, findings, and conclusions or recommendations expressed in this material are those of the authors and do not necessarily reflect the views of the National Aeronautics and Space Administration (NASA) or the USDA.

Conflict of Interest: The authors declare that the research was conducted in the absence of any commercial or financial relationships that could be construed as a potential conflict of interest.

Copyright (c) 2021 Zhen, van Iersel and Bugbee. This is an open-access article distributed under the terms of the Creative Commons Attribution License (CC BY).

The use, distribution or reproduction in other forums is permitted, provided the original author(s) and the copyright owner(s) are credited and that the original publication in this journal is cited, in accordance with accepted academic practice. No use, distribution or reproduction is permitted which does not comply with these terms. 